\title{
Anomalous persistence exponents for normal yet aging diffusion
}

\author{
A Barbier-Chebbah and O Benichou \\ Laboratoire de Physique Théorique de la Matière Condensée, \\ CNRS, UPMC, 4 Place Jussieu, 75005 Paris, France \\ R Voituriez \\ Laboratoire Jean Perrin, CNRS, UPMC, 4 Place Jussieu, 75005 Paris, France
}

(Dated: January 5, 2021)

\begin{abstract}
The persistence exponent $\theta$, which characterises the long time decay of the survival probability of stochastic processes in the presence of an absorbing target, plays a key role in quantifying the dynamics of fluctuating systems. So far, anomalous values of the persistence exponent $(\theta \neq 1 / 2)$ were obtained, but only for anomalous processes (i.e. with Hurst exponent $H \neq 1 / 2$ ). Here, we exhibit examples of aging processes which, even if they display asymptotically a normal diffusive scaling $(H=1 / 2)$, are characterised by anomalous persistent exponents that we determine analytically. Based on this analysis, we propose the following general criterion: the persistence exponent of asymptotically diffusive processes is anomalous if the increments display aging and depend on the observation time $T$ at all time scales.
\end{abstract}

The survival probability $S(t)$ of a 1-dimensional unbounded stochastic process $x(t)$ is defined as the probability that $x(t)$ has not reached a threshold value up to time $t$. This observable has proved to be very useful to quantify the dynamics of a broad range of complex systems in contexts as varied as diffusion controlled reactions, finance, search processes, or biophysics [15]. In many examples of symmetric stochastic processes, the large time behaviour of the survival probability is characterised by a power law decay $S(t) \propto t^{-\theta}$ that defines the persistence exponent $\theta$.

Because of its importance to characterise the dynamics of various systems, the determination of $\theta$ has been the focus of a vast amount of works in the fields of stochastic processes and non equilibrium statistical mechanics, and general results have been obtained in particular for processes without memory, i.e. Markovian processes [3, 4. More precisely, in the case of 1-dimensional, translation invariant symmetric Markovian jump processes, the Sparre Andersen theorem yields a robust universal result $\theta=1 / 2$, independently of the value of the Hurst exponent $H$ that characterises the large time behaviour of the mean square displacement $\left\langle x^{2}(t)\right\rangle \propto t^{2 H}$ [6]. In the case of processes with memory, i.e. non-Markovian processes, $\theta$ has been determined exactly only for specific examples of processes such as Fractional Brownian Motion [7, 8] or the random acceleration process [9], and perturbatively for weakly non Markovian Gaussian processes [10 12]. In all the examples studied so far, anomalous values of the persistence exponent ( $\mathrm{i}-\mathrm{e} \theta \neq 1 / 2$ ) were obtained, but only for anomalous processes (i.e. $H \neq 1 / 2$ ). In fact, all available examples of processes that are diffusive $(H=1 / 2)$, even if only asymptotically for $t \rightarrow \infty$, display the universal exponent $\theta=1 / 2$. This is illustrated by the example where $x(t)$ is the position of a given monomer of a finite 1-dimensional ideal Rouse chain of $N$ monomers ; this non Markovian process is diffusive at times larger than the Rouse time (that is the slowest relaxation time of the internal degrees of freedom of the chain) and is shown to be characterised by $\theta=1 / 2[5$; note that in this example the increments $x(t+T)-x(T)$ are stationary at long times, i.e. have statistics independent of the observation time $T$, for $T$ larger than the Rouse time. Other examples include persistent random walks and Levy walks and are discussed in [35]).

In this letter, we demonstrate that, in fact, anomalous persistence exponents can be obtained even for asymptotically normal diffusive processes. We argue that this results from the ageing properties of the process and propose the following general criterion: the persistence exponent of asymptotically diffusive processes is anomalous if the increments are non stationary and have statistics that depend explicitly on the observation time $T$ at all time scales. Our claim is based on the analysis of a range of examples of strongly non Markovian processes, and in particular of the so called Self Attracting Walk (SATW) [14] and the Elephant Random Walk (ERW) [15]. These have been studied in the context of random search processes as prototypical examples of processes with long range memory, and have important potential applications in the theoretical description of the trajectories of living organisms such as cells, insects or larger animals [1618. For both processes, we derive analytically the persistence exponent and show that it is anomalous, while we demonstrate that the processes are asymptotically diffusive, with however aging increments at all time scales. Our general criterion is confirmed by the analysis of further examples such as the persistent self attracting walk and the polynomial self repelling walk.

We consider first the so called Self Attracting Walk (SATW), or one-step reinforced walk, which was introduced as a natural example of random walk that interacts fully (either attractively or repulsively) with its own path [14, 19 25]. This model can therefore be related to the class of self-avoiding random walks, which have played a crucial role in physics [26], and have applications in the modelling of trajectories of living systems [27-29] ; in essence, such models consider a random walker that de- 
posits a signal at each visited site, which alters the future dynamics of the walker upon its next visits. Due to the long range memory effect of this class of processes, their properties, and in particular their persistence exponents, are notoriously difficult to characterise analytically. More precisely, the SATW is defined here on the $1 d$ lattice as a jump process in discrete time $t$ where the probability that the random walker jumps to a neighbouring site $i$ is proportional to $\exp \left(-u n_{i}\right)$, where $n_{i}=0$ if the site $i$ has never been visited up to time $t$, and $n_{i}=1$ otherwise. For $u>0$ the random walker is thus repelled by its own path, while it is attracted for $u<0$.



Figure 1. Definition of the SATW. The process is a simple nearest neighbor random walk in the bulk of the visited territory $\mathcal{D}_{t}$, and is modified only at the edges of $\mathcal{D}_{t}$; we show that increments $\left\langle[x(t+T)-x(T)]^{2}\right\rangle$ are diffusive at short and long time scales and display aging.

This process is strongly non-Markovian since the definition of its evolution at time $t$ requires the knowledge of the full territory $\mathcal{D}_{t}$ visited by the random walker up to time $t$ (see Fig1). However, in $1 d$, the knowledge of the two extrema $x_{-}=\inf _{0 \leq t^{\prime} \leq t} x\left(t^{\prime}\right)$ and $x_{+}=$ $\sup _{0 \leq t^{\prime} \leq t} x\left(t^{\prime}\right)$ of the trajectory at $t$ is sufficient to fully determine the visited territory $\mathcal{D}_{t}=\left[x_{-}, x_{+}\right]$and thus the evolution of the process. When in the bulk of the visited territory $\left(x_{-}<x(t)<x_{+}\right)$, the random walker performs a classical symmetric nearest neighbor random walk. The dynamics is modified only for $x=x_{-}, x_{+}$, and is then conveniently encoded locally by the probability $\beta=1 /(1+\exp (-u))$ to visit the yet unvisited neighboring site $\left(\notin \mathcal{D}_{t}\right)$ rather than the previously visited one $\left(\in \mathcal{D}_{t}\right)$. Only a few results are available to describe this non - Markovian process [14, 30 33, which has been shown in particular to be asymptotically diffusive at large times $\left\langle x^{2}(t)\right\rangle \propto t$, with however a large time diffusion coefficient that has not been determined analytically.

In view of determining the persistence exponent of this process, it is convenient to parametrise the dynamics in terms of the number $N=x_{+}-x_{-}+1$ of distinct sites visited up to time $t$. We define by $S(N)$ the corresponding survival probability that the target, located at $x=0$, has not been reached when $N$ sites have been visited ; in addition we denote by $x_{0}>0$ the initial position of the random walker. We first show below that $S(N)$ satisfies the following scaling in the large $N$ limit :

$$
S(N) \propto N^{-\frac{1-\beta}{\beta}},
$$

by providing lower and upper bounds for $S(N)$.
To this end, we note that a lower bound for $S(N)$ is straightforwardly given by the probability $P_{s}(N)$ that $\mathcal{D}_{t}=\left[x_{0}, x_{0}+N-1\right]$, i-e the probability that all of the $N$ visited sites have been discovered from the $x_{+}$edge of $\mathcal{D}_{t}$. Adapting classical results for splitting probabilities of $1 D$ random walks [1, 34] (see Supplementary Material for details [35]), an exact recurrence relation for $P_{s}(N)$ can be obtained and reads :

$$
P_{s}(N+1)=\left(1-\frac{1-\beta}{2+\beta(N-3)}\right) P_{s}(N) .
$$

Taking the $\log$ of Eq2 then yields in the large $N$ limit the following scaling of the lower bound :

$$
S(N)>P_{s}(N) \propto N^{-\frac{1-\beta}{\beta}} .
$$

We now turn to the determination of an upper bound to $S(N)$. We define a side change as the visit of a new site on the side opposite to the last newly visited site. With this definition, it is easy to see that all trajectories involving more than $2 x_{0}$ side changes cross the target at $x=0$. As a consequence, by defining $P(N, k)$ as the probability that exactly $k$ side changes occurred until $N$ sites have been visited in the presence of the target at $x=0$, one can write $S(N)=\sum_{k=0}^{2 x_{0}} P(N, k)$. In order to determine the scaling of $P(N, k)$, we introduce $k$ random variables $\left\{a_{1}, \ldots a_{k}\right\}$, where $a_{i}$ is the total number of distinct visited sites when the $i$ th side change occurs. Hence, between side changes $i$ and $i+1$, the random walker visits $a_{i+1}-a_{i}$ new sites on the same side of the visited territory $\mathcal{D}$. Correspondingly, we denote by $P_{s}\left(a_{i}, a\right)$ the probability that $a-a_{i}-1$ new sites have been visited on the same side of $\mathcal{D}$ after side change $i$. Note in particular that $P_{s}(1, N)=2 P_{s}(N)$ introduced above. We also introduce $P_{t}(a)$ as the probability that a side change occurs when the number of distinct visited sites is $a$; it is the probability that the random walker, starting from one edge of $\mathcal{D}$ discovers the next new site on the opposite edge.

The joint probability of the event $\left\{a_{1}, \ldots a_{k}\right\}$ can then be written

$$
\begin{aligned}
& P\left(\left\{a_{1}, \ldots a_{k}\right\}\right)=2^{-1} P_{s}\left(1, a_{1}\right) P_{t}\left(a_{1}\right) \\
& \quad \times\left(\prod_{i=2}^{k} P_{s}\left(a_{i-1}, a_{i}\right) P_{t}\left(a_{i}\right)\right) \times P_{s}\left(a_{k}, N\right) .
\end{aligned}
$$

Both $P_{s}$ and $P_{t}$ can be straightforwardly expressed in terms of splitting probabilities introduced above to determine $P_{s}(N)$, and exact recurrence relations similar to Eq. (2) can be obtained (see SM [35]). This yields in particular the following asymptotics $(i>1)$ :

$$
P_{s}\left(a_{i}, a_{i+1}\right) \underset{a_{i} \rightarrow \infty}{\sim} \frac{a_{i}^{\frac{1-\beta}{\beta}}}{a_{i+1}^{\frac{1-\beta}{\beta}}} ; \quad P_{t}\left(a_{i}\right) \underset{a_{i} \rightarrow \infty}{\sim} \frac{1-\beta}{\beta} \frac{1}{a_{i}},
$$

which will be useful below. In order to express $P(N, k)$, we must condition on the first step, and distinguish odd 
and even values of $k$. Assuming without loss of generality that the first step is made in the + direction, and that $k=2 p$ is even, we obtain in the large $N$ limit where sums can be approximated by integrals:

$$
\begin{aligned}
P(N, 2 p) \leq & \int_{1}^{N} \int_{a_{1}}^{\min \left(x_{0}+a_{1}, N\right)} \int_{a_{2}}^{N} \int_{a_{3}}^{\min \left(x_{0}+a_{3}, N\right)} . \\
& \int_{a_{2 p-2}}^{N} \int_{a_{2 p-1}}^{\min \left(x_{0}+a_{2 p-1}, N\right)} P\left(\left\{a_{1}, \ldots a_{k}\right\}\right) d a_{1} . . d a_{2 p} .
\end{aligned}
$$

Upper bounds in the integrals take into account the absorbing target at $x=0$. Next, using the definition of Eq. (4) and the asymptotics of Eq. (5), one obtains in the large $a_{i}$ limit

$$
P\left(\left\{a_{1}, \ldots a_{k}\right\}\right) \underset{a_{i} \rightarrow \infty}{\propto} N^{-\frac{1-\beta}{\beta}}\left(a_{1} a_{2} \times \ldots \times a_{k}\right)^{-1} .
$$

We then make use of the following estimate in the large $N$ limit derived in 35] :

$$
\begin{gathered}
\int_{1}^{N} \int_{a_{1}}^{\min \left(x_{0}+a_{1}, N\right)} \int_{a_{2}}^{N} . . \int_{a_{2 p-2}}^{N} \int_{a_{2 p-1}}^{\min \left(x_{0}+a_{2 p-1}, N\right)} \\
\left(a_{1} a_{2} \times . . \times a_{k}\right)^{-1} d a_{1} . . d a_{2 p}=O(1)
\end{gathered}
$$

to finally obtain

$$
P_{+}(N, 2 p)=O\left(N^{-\frac{1-\beta}{\beta}}\right) .
$$

The same $O\left(N^{-\frac{1-\beta}{\beta}}\right)$ estimate is obtained for $P_{ \pm}(N, k)$ for all combinations of the first step direction \pm and parity of $k$ as shown in SM 35. This finally yields

$$
S(N)=\sum_{k=0}^{2 x_{0}} P(N, k)=O\left(N^{-\frac{1-\beta}{\beta}}\right),
$$

which, together with the lower bound of Eq. (3) proves the scaling of Eq. (1). We finally conclude on the scaling of the survival probability $S(t)$ as a function of the elapsed time $t$. To do so, we make use of the ansatz that the conditional probability density $\Phi_{N}(t)$ that the target is found at time $t$ knowing that $N-1$ sites have been visited is a scaling function of the variable $t / N^{2}$ : $\Phi_{N}(t)=f\left(t / N^{2}\right) / N^{2}$ (see [35] for numerical check). This, together with Eq.(1), yields the following analytical determination of $\theta$ :

$$
\theta=\frac{1-\beta}{2 \beta}
$$

First, we underline that this result, which is expected to be exact (see numerical simulations Fig 2 shows that the SATW provides a simple model for which the persistence exponent is determined analytically and can be varied over a broad range of values by tuning a simple microscopic parameter $(\beta)$. Second, this reveals that,
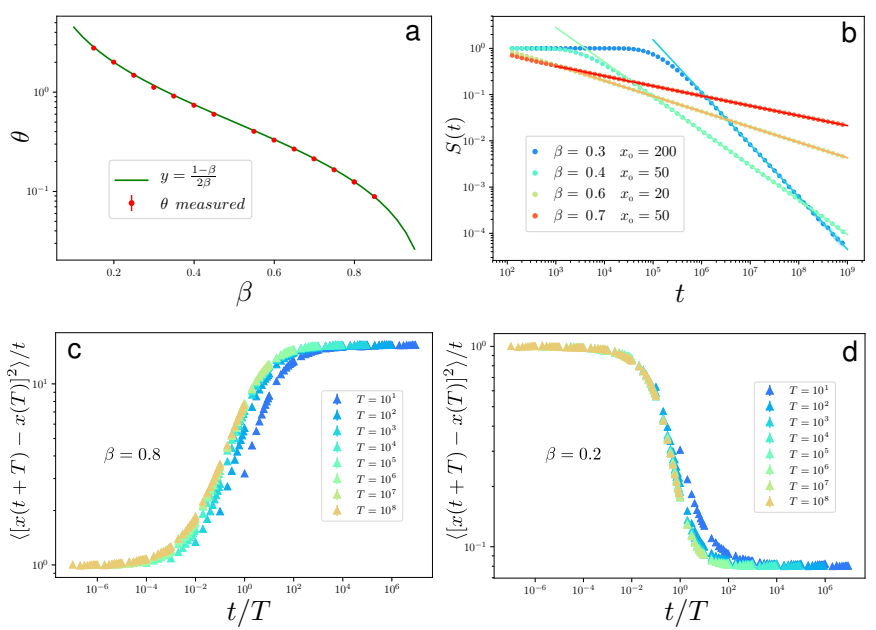

Figure 2. Persistence and aging of the SATW. a) Persistence exponent : numerical simulation and analytical prediction of Eq. 11. b) Survival probability as a function of time (log scale) for different values of $\beta$. Numerical simulations (symbols) and power law fit (plain line). c),d) Aging of the increments for the SATW (normalized by the expected diffusive scaling at long times). Each curve corresponds to a fixed value of $T$.

despite the fact that the process is asymptotically diffusive $\left\langle x^{2}(t)\right\rangle \propto t$ at large times $(H=1 / 2)$ for all values of $\beta$ [14, 30], the persistence exponent $\theta$ is anomalous $(\theta \neq 1 / 2)$ for $\beta \neq 1 / 2$.

Based on this example, we now aim at determining a general criterion that allows to identify the asymptotically diffusive processes that lead to anomalous persistence exponents. It has been proposed that for continuous scale invariant processes with stationary increments the universal relation $\theta=1-H$ should hold [4, 13]; this relation can in fact be extended to processes whose time dependent increments $\left\langle[x(t+T)-x(T)]^{2}\right\rangle$ are only asymptotically stationary after a finite characteristic timescale [5]. We review in 35] examples of such processes, which all lead to $\theta=1 / 2$. Our finding that $\theta \neq 1-H$ for the SATW indicates that its increments are not stationary after a finite time scale ; this calls for a further analysis of the increments. These are known to be diffusive and to satisfy $\left\langle[x(t+T)-x(T)]^{2}\right\rangle \sim 2 D_{L}(\beta) t$ in the large time limit $t \gg T$, where $D_{L}(\beta)$ has not been determined analytically. In turn it is easily seen that for $T \gg t$, one has $\left\langle[x(t+T)-x(T)]^{2}\right\rangle \sim 2 D_{s} t$ with $D_{s}=1 / 2$, because the walker spends most of the time away from the boundaries of the visited territory $\mathcal{D}_{T}$ (see Fig 1). Finally, because the process has no intrinsic time scale but the unit time step, dimensional analysis shows that in the limit $t, T \gg 1$ the increments can be written $\left\langle[x(t+T)-x(T)]^{2}\right\rangle=2 D(t / T) t$. This behavior was confirmed by numerical simulations (see Fig,2). Finally, the process is diffusive at both short and long time scales, but displays aging at all observation time scales $T$. We hypothesize that this behavior, which we call scale free aging, leads to anomalous persistence exponents for 
asymptotically diffusive processes. Below, we provide explicit examples supporting this hypothesis.

We first verify our hypothesis on a generalization of the SATW model to persistent random walks (see 35] for details). While the random walker is within the visited territory, it is now assumed to perform a classical persistent random walk of persistence probability (probability to reproduce the previous step) $p=\frac{e^{k}}{e^{k} e^{-k}}$, where $k$ is a free real parameter. When the random walker is at an edge $x_{ \pm}$of the visited domain, reinforcement and persistence effects are coupled so that the probability to discover a new site is given by $\beta(k, u)=\frac{e^{k}}{e^{-k-u}+e^{k}}$ (note that the SATW model is recovered by taking $k=0)$. It is shown in [35, that the persistence exponent can still be determined explicitly for this model ; it is found to be independent of $k$ :

$$
\theta=\frac{1-\beta(k=0, u)}{2 \beta(k=0, u)} .
$$

As in the SATW case $(k=0)$, the persistence exponent is thus anomalous for $\beta(k=0, u) \neq 1 / 2$. The analysis of the increments reveals that motion is ballistic below the time scale $e^{2 k}$. In turn, for $T, t \gg e^{2 k}$, increments are characterized by a time dependent diffusion coefficient $D(t / T)$ that ranges from $D_{s}=e^{2 k} / 2$ for $T \gg t$ to an undetermined ( $k$ dependent) value $D_{L}$ for $t \gg T$ (see 35]). The process therefore displays scale free aging, and our hypothesis is verified. To further assess the generality of our criterion, we analyse below two other examples of aging asymptotically diffusive non Markovian processes with anomalous persistence exponents.

First, the so-called elephant random walk (ERW) was introduced in 15, and can be defined as follows on $1 d$ lattice : at each time step $t$ a nearest neighbor jump $\bar{\sigma}$ is drawn with uniform distribution from the full set of jumps performed in the past $\left\{\sigma_{i}\right\}_{i<t}$, where $\sigma_{i}= \pm 1$ (with a symmetric first jump). The random walker then performs the jump $\sigma_{t}=\bar{\sigma}$ with probability $\beta$, and the jump $\sigma_{t}=-\bar{\sigma}$ with probability $1-\beta$. The position process is then defined by $x(t)=\sigma_{t}+x(t-1)$. The process is non Markovian by construction, and can be shown for $\beta<3 / 4$ to be characterized by scale free, diffusive, aging increments $\left\langle[x(t+T)-x(T)]^{2}\right\rangle \sim 2 D(t / T) t$ in the limit $T, t \gg 1$, with an effective diffusion coefficient that we show can be written (see [35])

$$
D(t / T) \sim \frac{1}{6-8 \beta}-\frac{1}{3-4 \beta}\left[\frac{T}{t}\left(\left(1+\frac{t}{T}\right)^{2 \beta-1}-1\right)\right] .
$$

This behavior of the increments (see Fig 3) is similar to the case of the SATW, with in particular finite limits of the effective diffusion coefficient $D(t / T)$ in both regimes $t \ll T$ and $t \gg T$ (see Fig 3 ). The limit diffusion coefficients can be expressed analytically from 13 and read $D_{s}=1 / 2$ and $D_{L}=1 /(6-8 \beta)$ (in agreement with [15, 36] for $T=0)$. Similarly to the SATW, the ERW is therefore diffusive at both short and time scales, and displays scale free aging. We now determine the persis-
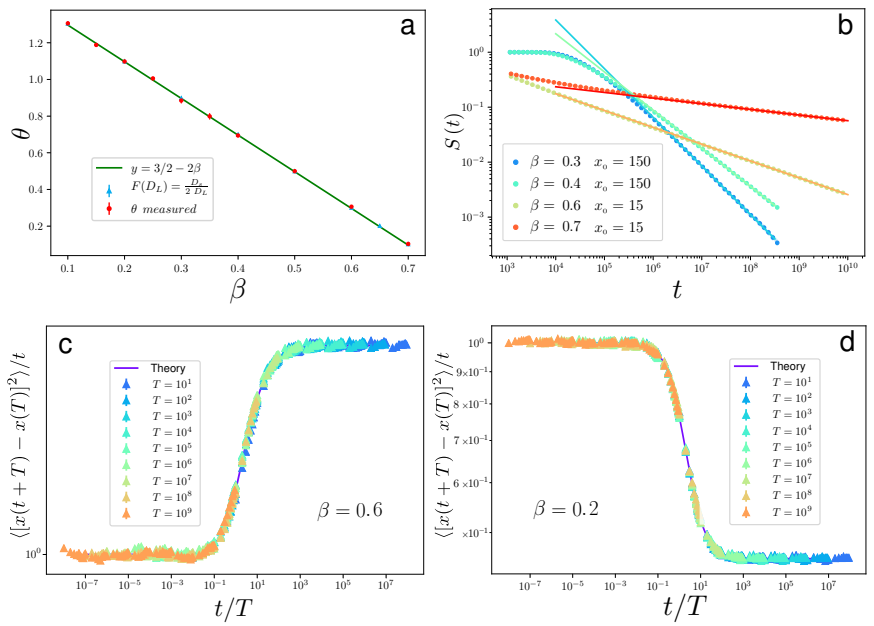

Figure 3. Persistence and aging of the ERW. a) Persistence exponent : numerical simulations, analytical prediction of Eq. 14 and prediction based on an effective Fick's law 35.

. b) Survival probability as a function of time (log scale) for different values of $\beta$. Numerical simulations (symbols) and power law fit (plain line). c),d) Aging of the increments for the ERW (normalized by the expected diffusive scaling at long times) and analytical prediction of Eq.13 (plain line).

Each curve corresponds to a fixed value of $T$.

tence exponent of the ERW. In the diffusive regime that we consider here, it has been shown that in the large $t$ limit, the 1-point probability density function $p(x, t)$ is Gaussian [36] and satisfies a Fokker-Planck equation with a time-dependent drift for $\beta<3 / 4$; based on this formalism, the first-passage time distribution to a target was computed in [37]. We used this result to derive analytically the persistence exponent of the ERW:

$$
\theta=3 / 2-2 \beta,
$$

which was confirmed by numerical simulations (see Fig.32. Again, we find that the persistence exponent $\theta$ is anomalous $(\theta \neq 1 / 2)$ for $\beta \neq 1 / 2$, and takes a broad range of values when $\beta$ is varied. The example of the ERW therefore supports our conjecture that scale free aging is responsible for the observed anomalous persistent exponent $\theta \neq 1 / 2$, even for asymptotically diffusive processes $(H=1 / 2)$.

Last, we consider an example of self-repelling walk where the probability that the random walker jumps to a neighbouring site $i$ is proportional to $1 /\left(1+\tau_{i}^{\beta}\right)$, where $\tau_{i}$ is defined as the cumulative time spent by the random walker at the site $i$. This process has been shown to be asymptotically diffusive at large times [38, 39] ; however its increments and persistence properties remain unexplored. Our numerical analysis reveals that the persistence exponent is anomalous $\theta \approx 0.25$ for all values of $\beta$ ; in turn the increments are found to display scale free aging (see 35]). Our hypothesis is therefore verified once again. 
We now conclude this letter by summarizing our results. We have analysed the persistence exponents for several examples of Non-Markovian random walks, which have been studied in the context of random search processes. These persistence exponents, some of which we determined analytically, were found to be anomalous, and to vary over a broad range of values even if all processes remain asymptotically simply diffusive. Our analysis leads to the conjecture that the persistence exponent of asymptotically diffusive processes is anomalous if the increments are aging and depend on the observation time $T$ at all time scales.
[1] S. Redner, A Guide to First- Passage Processes (Cambridge University Press, Cambridge, England, 2001).

[2] R. Metzler, G. Oshanin, and S. Redner, First passage problems: recent advances (World Scientific, Singapore, 2014).

[3] S. N. Majumdar, Curr. Sci. 77, 370 (1999).

[4] A. J. Bray, S. N. Majumdar, and G. Schehr, Advances in Physics, Advances in Physics 62, 225 (2013).

[5] N. Levernier, M. Dolgushev, O. Bénichou, R. Voituriez, and T. Guérin, Nat Commun 10, 2990 (2019).

[6] E. S. Andersen, Mathematica Scandinavica 2, 195 (1954).

[7] J. Krug, H. Kallabis, S. N. Majumdar, S. J. Cornell, A. J. Bray, and C. Sire, Physical Review E 56 (1997).

[8] G. Molchan, Communications In Mathematical Physics 205, 97 (1999).

[9] T. W. Burkhardt, Journal of Physics A: Mathematical and General, 26, L1157 (1993)

[10] Majumdar and Sire, Phys Rev Lett 77, 1420 (1996).

[11] S. N. Majumdar and A. J. Bray, Physical Review Letters 81, $2626(1998)$

[12] K. J. Wiese, S. N. Majumdar, and A. Rosso, Physical Review E 83 (2011)

[13] A. Zoia, A. Rosso, and S. N. Majumdar, Physical Review Letters 102, 120602 (2009)

[14 V. B. Sapozhnikov, Journal of Physics A: Mathematical and General 27, L151 (1994)

[15] G. M. Schütz and S. Trimper, Physical Review E 70, 045101 (2004)

[16] D. Boyer and P. D. Walsh, Philosophical Transactions of the Royal Society A: Mathematical, Physical and Engineering Sciences 368, 5645 (2010).

[17] D. Boyer, M. C. Crofoot, and P. D. Walsh, Journal of The Royal Society Interface 9, 842 (2012), publisher: Royal Society.

[18] L. Börger, B. D. Dalziel, and J. M. Fryxell, Ecology Letters 11, 637 (2008)

[19] R. Pemantle, Probability Surveys 4, 1 (2007)

[20] D. J. Amit, G. Parisi, and L. Peliti, Physical Review B 27, 1635 (1983), publisher: American Physical Society.
[21] L. Peliti and L. Pietronero, La Rivista del Nuovo Cimento (1978-1999) 10, 1 (1987)

$[22]$ J. G. Foster, P. Grassberger, and M. Paczuski, New Journal of Physics 11, 023009 (2009), publisher: IOP Publishing.

[23] A. Ordemann, E. Tomer, G. Berkolaiko, S. Havlin, and A. Bunde, Physical Review E 64, 046117 (2001)

[24] M. Perman and W. Werner, Probability Theory and Related Fields 108, 357 (1997)

[25] B. Davis, Probability Theory and Related Fields 84, 203 (1990)

[26] P. G. de Gennes, Scaling Concepts in Polymer Physics (Ithaca, 1979).

[27] A. Stevensand H. G. Othmer, SIAM Journal on Applied Mathematics 57, 1044 (1997)

[28] D. Boyer and J. C. R. Romo-Cruz, Physical Review E 90, 042136, (2014).

[29] H. Freund and P. Grassberger, Physica A: Statistical Mechanics and its Applications 190, 218 (1992)

[30] M. A. Prasad, D. P. Bhatia, and D. Arora, Journal of Physics A: Mathematical and General 29, 3037 (1996)

[31] B. Davis, Annals of Probability 24, 2007 (1996), publisher: Institute of Mathematical Statistics.

[32] E. Agliari, R. Burioni, and G. Uguzzoni, New Journal of Physics 14, 063027 (2012)

[33] P. Carmona, F. Petit, and M. Yor, Journal of the London Mathematical Society 58, 239 (1998).

[34] N. Van Kampen, Stochastic Processes in Physics and Chemistry, Third Edition (North-Holland personnal library, 1992).

[35] See the Supplemental Material .

[36] C. F. Coletti, R. Gava, and G. M. Schütz, Journal of Mathematical Physics 58, 053303 (2017).

[37] A. T. Silva, E. K. Lenzi, L. R. Evangelista, M. K. Lenzi, H. V. Ribeiro, and A. A. Tateishi, Journal of Mathematical Physics 52, 083301 (2011)

[38] B. Tóth (Birkhaüser Basel, 2001) pp. 555-564.

[39] B. Tóth, The Annals of Probability 24, 1324 (1996), publisher: Institute of Mathematical Statistics. 